\title{
Charge Aborted
}

National Cancer Institute

\section{Source}

National Cancer Institute. Charge Aborted. NCI Thesaurus. Code C63281.

Problem associated with the premature ending of the charging process (e.g. of a battery or other charge storage device). 